BANCODEESPAÑA

Eurosistema

\title{
Artificial intelligence in financial services
}

Ana Fernández 


\section{Abstract}

The use of artificial intelligence tools has escalated recently in all sectors of the economy owing, among other factors, to the growing volume of digital data and higher computational capacity. Major benefits may be reaped from applying these tools to the provision of financial services, not only for financial institutions but also for society as a whole. This article describes some of those benefits, and also some of the main uses being made of these tools, both by financial institutions and central banks. It also indicates the main limitations of the technology and its possible implications for the correct functioning of the financial system.

Keywords: artificial intelligence, machine learning, algorithm, automation, chatbots, bias. JEL codes: $031,033$. 


\section{ARTIFICIAL INTELLIGENCE IN FINANCIAL SERVICES}

The author of this article is Ana Fernández, of the Directorate General Operations, Markets and Payment Systems. ${ }^{1}$

Introduction

What is artificial intelligence?

Benefits of artificial intelligence
Although artificial intelligence dates back more than half a century, its possibilities have increased significantly in recent times. This is prompting the development of numerous practical applications, both in the financial and other sectors.

Artificial intelligence tools can provide major benefits, allowing certain tasks to be automated and boosting analytical capacity compared with traditional techniques. However, they also present a series of limitations that can make them unsuitable for certain activities, along with a set of risks that must be managed appropriately.

The following paragraphs describe in more detail the use financial institutions are making of these tools, together with some of the opportunities and challenges they present.

Drawing on the definition coined by the Financial Stability Board (FSB), ${ }^{2}$ artificial intelligence is the set of theories and algorithms that allow computer systems to perform tasks which typically require human intelligence (e.g. visual perception, voice recognition, or interpretation of a text taking into account its context) and that in some cases augment these skills.

Most current artificial intelligence applications belong to the field of machine learning. This consists in a computer drawing conclusions from a statistical analysis of data, in a process that improves automatically as more and more information is fed into the algorithm.

Artificial intelligence is not a new development; indeed the first academic studies date back to the 1950s. However, it has gained popularity recently, owing mainly to three factors: the growing volume of digital data available; increased data storage and computational processing capacity and its lower cost; and the progress made in the algorithms used. Thanks to these changes, better use can now be made of the capacities of artificial intelligence, and in consequence its use is growing significantly, not only in the financial sector but also in the economy overall (see Chart 1).

However, this level of development differs between regions. According to figures published by the European Commission in December 2018, ${ }^{3}$ private investment in artificial intelligence amounts to more than $€ 6.5$ billion in Asia and more than $€ 12$ billion in North America, whereas in Europe it is less than $€ 3.5$ billion. This is partly due to the privileged position enjoyed by the Asian and US technology giants for developing artificial intelligence tools, as they have both the infrastructure and the necessary data at their disposal. Accordingly, these corporations make intensive internal use of this technology, and they have also become leading global suppliers of artificial intelligence applications (e.g. image recognition, translation, natural language processing, etc.).

Using artificial intelligence techniques in the provision of financial services can heighten efficiency, reduce costs, enhance quality, raise customer satisfaction levels and boost

1 The author wishes to thank Juan Ayuso, Carlos Conesa, José Manuel Marqués, Sergio Gorjón, Manuel Ortega, Galo Nuño and her colleagues in the Financial Innovation Division for their comments.

2 See Financial Stability Board (2017).

3 See European Commission (2018). 
Venture capital financing for start-ups specialising in artificial intelligence applications has increased continuously since 2012, tripling between 2016 and 2017 .

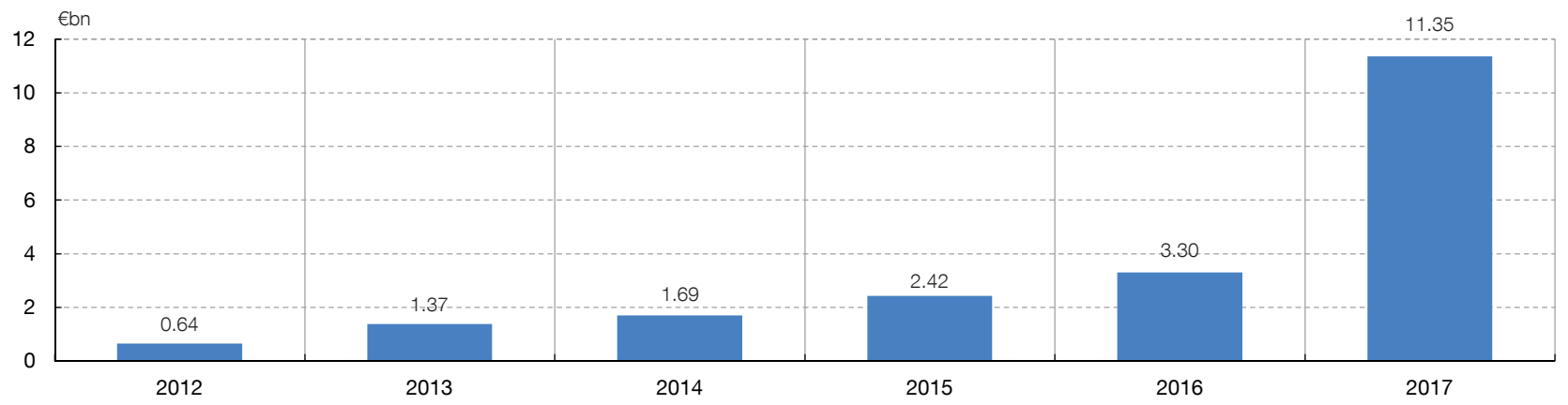

SOURCE: European Commission (2018).

Download

GREATER AUTOMATION

GREATER ANALYTICAL CAPACITY

Examples of use in financial services financial inclusion, mainly thanks to the possibilities they offer for automating operating processes and increasing analytical capacity.

Techniques such as natural language processing or image recognition allow financial institutions to automate repetitive or low added-value tasks (e.g. replies to FAQs). This reduces the potential for human error, boosts productivity and cuts the cost of these tasks.

As a result customer satisfaction levels rise, since customers receive better service (shorter response time and greater availability of services) and, potentially, for a lower price. Also, given the cost savings resulting from task automation, this may make it possible to offer certain services that were traditionally reserved for certain customers (e.g. financial advice) to a broader range of users.

Thanks to artificial intelligence tools, financial institutions are able to analyse a much higher volume of both structured and unstructured data much faster. In addition, the increased number of variables boosts the quality of the analysis, as knowledge of customers is greater and the results obtained are more precise.

All the above is to the advantage of users, as it allows financial institutions to offer better service (e.g. greater accuracy in detecting card fraud) and grants access to financial services to certain customers who might otherwise have been excluded (e.g. lending to customers with whom a bank had no previous relationship and on whom there is no financial information). It may also provide substantial cost savings in areas, such as regulatory compliance, where recent developments are putting pressure on costs.

In addition, greater analytical capacity allows financial institutions to exploit all the data (both internal and external) to which they have access on their customers, to learn more about their preferences. In this way they are able to develop tailor-made products and services, and even anticipate their customers' needs, improving user experience (e.g. by offering home insurance when they detect that customers are considering buying a new home).

As Figure 1 shows, numerous uses may be found for artificial intelligence in the different areas of the financial sector, across the entire value chain, from back-office through middle-office to front-office. Some applications aim to improve customer experience 
Financial institutions are applying artificial intelligence tools across the entire value chain and to all kinds of services.

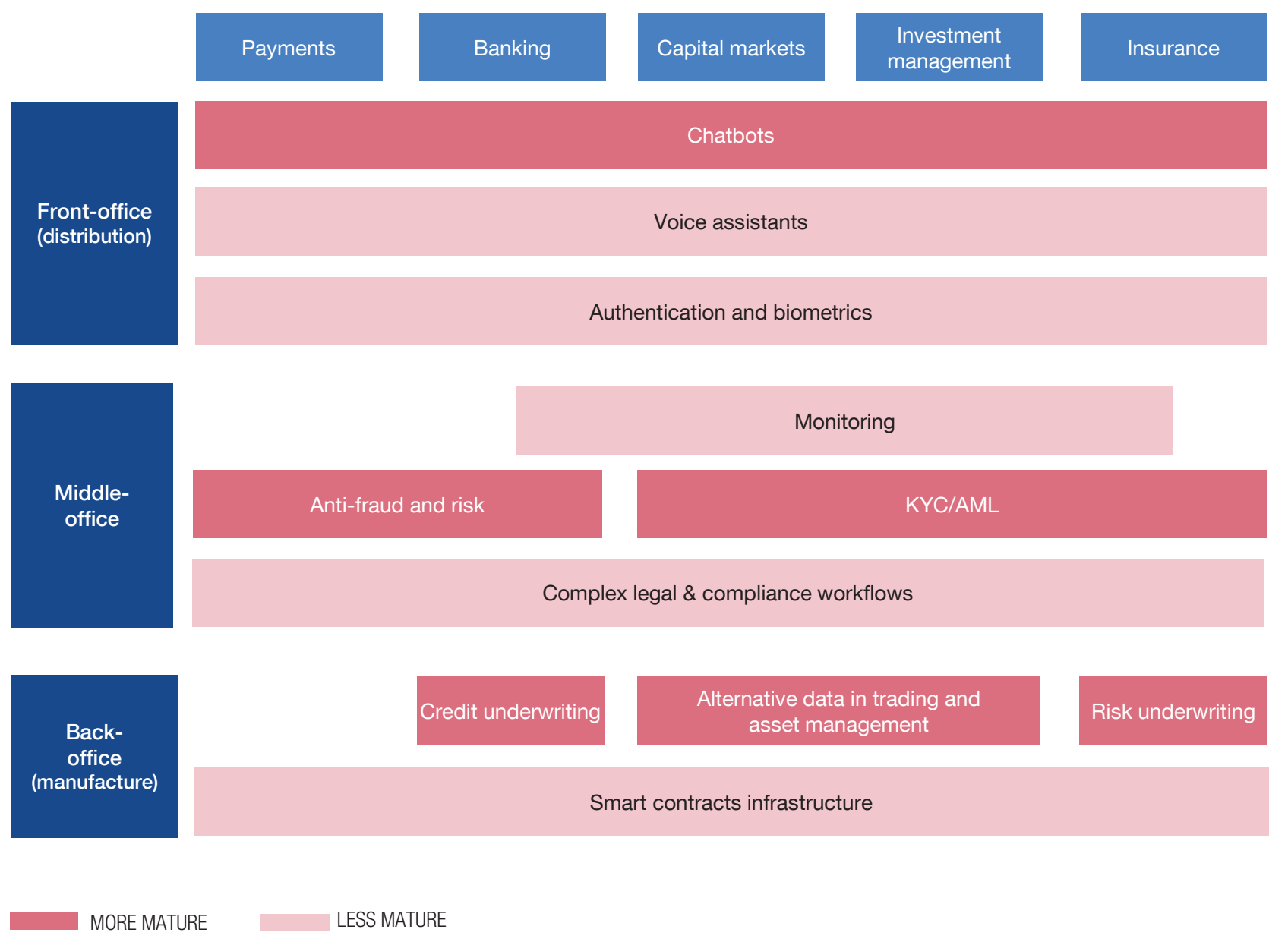

SOURCE: Autonomous NEXT.

(e.g. biometric authentication), while others seek to optimise and/or transform processes in the different stages of the provision of financial services (e.g. using new data sources to select investments or determine risk premia). Combining artificial intelligence with other technologies (such as distributed ledger technology and smart contracts) may potentially increase the possibilities even further.

Without seeking to be exhaustive, the following examples of the use of artificial intelligence in the financial sector may be highlighted: ${ }^{4}$

- Chatbots and virtual assistants. These enable users to settle common doubts ${ }^{5}$ and, in some cases, to secure product recommendations or perform certain transactions (e.g. order transfers, open accounts). Communication channels with customers thus become automated, which means that they are available $24 / 7$ and, in addition, that data on interactions with users are collated automatically.

4 For more information, see Institute of International Finance (2016).

5 By means of written or (in the case of voice assistants) spoken conversations. 
- Customisation of end products and services. Based on the information obtained from customers, financial institutions are able to offer a personalised user experience, possibly even beyond banking services in the strictest sense of the term (e.g. sending account balance alerts if geolocalisation services detect that customers are shopping).

- Control of anti-money laundering (AML) and fraud prevention. The ability to analyse a greater volume of data and to combine those data with new sources of information enables anomalies or patterns to be detected that would otherwise have gone unnoticed. ${ }^{6}$ It also results in a lower number of false positives, which makes for faster and more accurate fraud and AML control tasks and less inconvenience to customers.

- Credit scoring. The increased analytical capacity can improve credit assessment and make loan origination faster. In addition, the use of unstructured data can boost the number of eligible customers, by facilitating this analysis to new financial institutions or to those acting in new markets on which there is little information available.

- Regulatory compliance. The greater analytical capacity that artificial intelligence tools provide eases compliance with certain regulatory requirements (e.g. risk management, reporting obligations) and also monitoring of regulatory changes.

\section{Applications in central banks}

\section{Main limitations}

Authorities may also use artificial intelligence to their advantage in terms of operating efficiency and cost savings. Accordingly, various central banks are beginning to incorporate it into some of their activities, ${ }^{7}$ such as microprudential and macroprudential supervision, information management, forecasting, or detecting fraudulent activity. For example, the Monetary Authority of Singapore is developing a tool for analysis of reports on suspicious transactions, while the central bank of Austria has developed a prototype for data validation. The central bank of Italy is using artificial intelligence techniques to predict price moves on the real estate market, and the central bank of the Netherlands to anticipate potential liquidity problems at financial institutions.

At the Banco de España, an artificial intelligence tool has been developed for sorting banknotes between fit and unfit for circulation. In addition, statisticians are analysing how artificial intelligence may be used to improve the quality control of the information available in micro-databases (specifically, annual accounts deposited by non-financial corporations), while economists are assessing the extent to which these techniques could enhance macroeconomic analysis models and indicator selection. ${ }^{8}$

Despite the significant benefits that artificial intelligence offers, it also comes with a series of limitations that must be taken into account when assessing its use. The main risks stem from the potential bias in the results obtained using these tools, and from the difficulties involved in understanding the reasoning process followed by the algorithms to reach a specific conclusion.

6 See Van Liebergen (2017).

7 See Bank for International Settlements (2018).

8 For more details, see Fernández-Villaverde et al. (2019), García-Uribe (2018), Ghirelli et al. (2019) and Gil et al. (2018). 
Essentially, algorithms operate by seeking correlations that will maximise predictive power. In some cases, this can produce results based on spurious relationships and thus lead to biased conclusions being drawn. ${ }^{9}$ How important this bias is will depend, naturally, on the context in which it arises. Bias in translation, for example, is not the same as bias in loan origination. In any event, it is important to know why this bias occurs.

Unintentional bias may stem from the data analysed or from the way in which the algorithm is trained. Algorithms must be trained with a huge volume of quality data, i.e. data that are representative of the total population. Otherwise, any bias in the training sample may become a criterion to be met and thus an obstacle to equal opportunity (for example, in the case of job selection processes or loan origination).

Moreover, unwanted bias may arise from the way in which algorithms are designed or operate (e.g. from the labelling of the training data or from how the algorithms evolve as they include new information). One example of this was the experimental chatbot that a technology company launched on a social network in order for it to learn by chatting with users and that was rapidly withdrawn in view of the inappropriate content its messages had acquired.

INTERPRETATION OF ALGORITHMS

\section{Challenges for financial authorities}

Algorithms generally operate in a complex way and this makes it difficult to understand the reasoning they follow to reach a specific outcome. Although in some cases it may not be important to understand this process (a chatbot, for example), in others it may be necessary to explain why a specific decision has been taken (for example, in credit scoring). Clearly it is not a question of knowing the entire process followed by the algorithm to reach a specific conclusion, but it would seem reasonable to establish a set of requirements to ensure the visibility of the process whereby a specific conclusion is reached.

Work is currently under way on developing techniques to heighten the transparency of algorithms, ${ }^{10}$ but the results are still difficult to interpret. The possible implications for the use of applications for regulatory compliance ${ }^{11}$ or supervisory-related activities (for example, assessing credit risk models using artificial intelligence tools) are more than evident.

In addition to the problems deriving from the limitations analysed above, authorities also need to consider other potential risks relating to the market in which artificial intelligence applications are developed: ${ }^{12}$

- Concentration risk. Developing artificial intelligence tools requires a large volume of data and extensive resources. This encourages concentration among a small number of large players, with a potentially adverse impact on competition. Requirements in terms of access to third-party data, such as those envisaged in the new Payment Services Directive (PSD2), could ease this problem.

- Systemic risk. A high level of reliance on tools and infrastructures provided by technology companies for use in operations deemed critical could

9 See Petrasic et al. (2017).

10 See Institute of International Finance (2018).

11 In this respect, the General Data Protection Regulation recognises the right to receive, in certain cases, information on the logic involved in the case of automated decisions based on personal data processing. See Goodman and Flaxman (2016).

12 See Wall (2018). Another key challenge to be considered, although one that is beyond the scope of this article, is the impact of artificial intelligence on the labour market. 
increase financial institutions' operational risk and, given the small number of providers, could lead to systemic risk. In addition, the widespread use of algorithms of this kind in activities such as the provision of credit or trading of financial assets could lead to herding and, eventually, procyclical behaviour. However, whether or not this risk materialises will depend, in the end, on the degree of uniformity of the data used by financial institutions and of the training mechanisms and methodologies employed to develop the algorithms.

- Customer protection. The need to use such a huge volume of data heightens the significance of privacy issues and increases the risk of some financial institutions using certain data without their customers being fully aware of this. Doubts also arise regarding which party would be liable (the financial institution or the algorithm provider) for any loss resulting from the use of artificial intelligence techniques.

Authorities also face a series of challenges and limitations when it comes to determining whether or not to use artificial intelligence tools in the pursuit of their own functions. ${ }^{13}$ In addition to the challenge of finding staff with expertise in this field, there is the fact that the capacity of artificial intelligence applications to detect possible problems at financial institutions or signs of crisis on the markets depends, as indicated above, on the quality and breadth of the data used to train the algorithms. Nevertheless, most of those data relate to normal rather than crisis situations.

\section{Conclusions}

13 See Danielsson et al. (2017). basis. provided by algorithms.

The use of artificial intelligence applications in the financial sector is already a tangible reality that provides numerous benefits to market participants. However, it also poses a series of risks and limitations that must be known and managed so as to be able to correctly extract all their potential. To that end, a distinction must be drawn between the different types of activities for which these tools are used, since problems such as results bias or difficulties interpreting the underlying reasoning are more important in some areas than in others. Accordingly, the advantages and drawbacks of using this technology, along with the way in which it should be used, need to be assessed on an individual case

One option which is relatively common and which in most cases offers a reasonable balance between risks and rewards is to use artificial intelligence tools together with (rather than instead of) traditional techniques. In other words, to use these tools as part of a process to reinforce and validate decisions, rather than accepting outright the results

Given the growing proliferation of artificial intelligence-based solutions, and considering their possible implications, the Banco de España will continue to monitor their use in the financial sector and all new developments. Cooperation with other authorities, both at a national and a global level, is particularly important in this respect, given that the use of artificial intelligence crosses both national and sectoral boundaries. 
BANK FOR INTERNATIONAL SETTLEMENTS (2018). Innovative technology in financial supervision (suptech) - the experience of early users, FSI Insights on policy implementation, No 9.

DANIELSSON, J., R. MACRAE and A. UTHEMANN (2017). Artificial intelligence, financial risk management and systemic risk, Systemic Risk Centre Special Paper, SP 13.

EUROPEAN COMMISSION (2018). The Age of Artificial Intelligence, EPSC Strategic Notes, Issue 29.

FERNÁNDEZ-VILLAVERDE, J., S. HURTADO and G. NUÑO (2019). Financial Frictions and the Wealth Distribution, mimeo.

FINANCIAL STABILITY BOARD (2017). Artificial intelligence and machine learning in financial services.

GARCÍA-URIBE, S. (2018). The effects of tax changes on economic activity: a narrative approach to frequent anticipations, Banco de España Working Paper 1828.

GHIRELLI, C., J. J. PÉREZ and A. URTASUN (2019). A new economic policy uncertainty index for Spain, Banco de España Working Paper 1906.

GIL, M., J. J. PÉREZ, A. J. SÁNCHEZ and A. URTASUN (2018). Nowcasting private consumption: traditional indicators, uncertainty measures, credit cards and some internet data, Banco de España Working Paper 1842.

GOODMAN, B. and S. FLAXMAN (2016). European Union regulations on algorithmic decision-making and a "right to explanation".

INSTITUTE OF INTERNATIONAL FINANCE (2016). Digitizing intelligence: Al, robots and the future of finance.

- (2018). Explainability in predictive modeling, Machine Learning Thematic Series Part I.

PETRASIC, K., B. SAUL, J. GREIG, M. BORNFREUND and K. LAMBERTH (2017). Algorithms and bias: What lenders need to know.

VAN LIEBERGEN, B. (2017). "Machine Learning: A Revolution in Risk Management and Compliance?", The CAPCO Institute Journal, No 45.

WALL, L. (2018). "Some Financial Regulatory Implications of Artificial Intelligence", Journal of Economics and Business, vol. 100 , pp. 55-63. 